\title{
CLOSURES OF WEAKENED ANALYTIC GROUPS
}

\author{
T. CHRISTINE STEVENS
}

(Communicated by Jonathan M. Rosenberg)

\begin{abstract}
Let $(G, \mathscr{G})$ be a topological group with dense subgroup $L$, and suppose that $L$ is an analytic group in a topology $\tau$ that is stronger than the topology that $L$ inherits from $\mathscr{G}$. It is known that $L$ contains a $\tau$-closed abelian subgroup $H$ that completely determines the topology of $L$. We now prove that the $\mathscr{G}$-closure $\bar{H}$ of $H$ similarly determines the topology of $G$. $(G, \mathscr{G})$ always has a left-completion in the category of topological groups, and the properties of $\bar{H}$ determine whether $(G, \mathscr{G})$ is locally compact, analytic, metrizable, left-complete, or finite dimensional. We discuss the relationship between these results and recent work of Goto.
\end{abstract}

\section{INTRODUCTION}

Analytic groups with the "wrong" topology-that is, with a topology weaker than the usual one-arise in several contexts, including the study of immersions of analytic groups, and have been investigated by Goto [4] and by the author [9, $10]$. In this paper we extend our previous work by examining topological groups $G$ in which a "weakened analytic" group $L$ is dense and showing that the topology of such a group is completely determined by the closure of a certain abelian subgroup of $L$. This subgroup then determines whether $G$ is locally compact, metrizable, analytic, complete, or finite dimensional. Moreover, $G$ always has a left-completion in the category of topological groups. These theorems, which resemble but extend beyond recent results of Goto [4], are stated precisely in $\S 3$. The first step in the proof is the construction in $\S 4$ of a specific completion of $L$, which is then shown to be a completion of $G$ as well. In $\S 5$ we analyze the topology of $G$ by studying how it "sits" in this completion. Section 6 discusses in detail the relationship between Goto's work and ours, and in $\S 7$ we close with a conjecture that affects the scope of our results.

\section{NOTATION AND DEFINITIONS}

Topologies for abstract groups are assumed to be Hausdorff and to make the group operations continuous. If $(G, \mathscr{G})$ is a topological group and $A$ is a subgroup of $G$, then $\mathscr{G}_{A}$ denotes the topology that $A$ inherits from $G$. If $(L, \tau)$ is an analytic (i.e., connected Lie) group and $\mathscr{U}$ is a topology for $L$

Received by the editors June 14, 1991; presented to the Society on January 15, 1992.

1991 Mathematics Subject Classification. Primary 22A05; Secondary 22E15.

Key words and phrases. Analytic group, weakened analytic group, completion of a group. 
that is weaker than $\tau$, then $(L, \mathscr{U})$ is a weakened analytic (WA) group. When different topologies for the same abstract group are under simultaneous consideration, topological statements about that group will mention the particular topology involved.

The topological group $(G, \mathscr{G})$ is complete if it is complete in its left uniform structure $\mathscr{L}(\mathscr{G})$; in that case, of course, $G$ is also complete in its right and two-sided uniform structures. If $G$, with its left uniform structure, has a completion in the category of topological groups, we say simply that $(G, \mathscr{G})$ has a completion.

Suppose the abstract group $G$ contains subgroups $A$ and $B$ such that $G=$ $A B$ and $A$ is normal in $G$. We now describe a technique that generalizes slightly a notion introduced in $[10, \S 3]$ and enables us to combine topologies for $A$ and $B$ into a topology for $G$. Let $I(b)$ denote conjugation by an element $b$ of $B$, and let $\mathscr{A}$ and $\mathscr{B}$ be topologies for $A$ and $B$, respectively, that make the evaluation map $f: A \times B \rightarrow A$, defined by $f(a, b)=b a b^{-1}$, $(\mathscr{A} \times \mathscr{B}, \mathscr{A})$-continuous. Then $\mathscr{A} \times \mathscr{B}$ is a group topology for the semidirect product $A(S B$, and we can define a surjective homomorphism $\alpha: A(B \rightarrow G$ by $\alpha(a, b)=a b$. If $A \cap B$ is $\mathscr{A}$-closed and inherits a weaker topology from $\mathscr{B}$ than from $\mathscr{A}$, then the unique topology for $G$ that makes $\alpha$ open and continuous is a (Hausdorff) group topology for $G$, which is called the standard extension of $\mathscr{A}$ and $\mathscr{B}$ and is denoted by $\mathscr{E}(\mathscr{A}, \mathscr{B})$.

\section{Main Results}

To facilitate the statement of our principal theorems, we first summarize for easy reference the properties of (WA) groups that will be used in this paper.

Theorem 3.1 [9, Theorem 3.1]. Let $(L, \mathscr{U})$ be a $(W A)$ group, with $\tau$ the analytic topology for $L$ and $\mathbf{1}$ its Lie algebra. Then $L$ contains a $\tau$-closed abelian subgroup $H$, which depends on $(L, \tau)$ but not on $\mathscr{U}$, with the following properties:

(i) The function $I: H \rightarrow \operatorname{Aut}(L)$, which assigns to each $x \in H$ the inner automorphism of $L$ by $x$, is $\mathscr{U}_{H}$-continuous if $\operatorname{Aut}(L)$ is given the generalized compact-open topology.

(ii) $\mathscr{U}=\mathscr{E}\left(\tau, \mathscr{U}_{H}\right)$.

(iii) If $(L, \tau)$ is $(C A)$-that is, if the adjoint image of $L$ is closed in the general linear group $\mathrm{GL}(\mathbf{l})$-then $H$ is the center $Z(L)$ of $L$.

(iv) If $(L, \tau)$ is not $(C A)$, then $H=V \times Z(L)$, where $V$ is a vector group.

$H$ is called a decisive subgroup of $(L, \tau)$. The existence of decisive subgroups is proved in [10] and their structure is further investigated in [9].

Throughout the remainder of this paper, $(G, \mathscr{G})$ will denote a topological group in which a (WA) group $\left(L, \mathscr{G}_{L}\right)$ is dense. Let $\tau$ be the analytic topology for $L$ and $H$ the abelian decisive subgroup of Theorem 3.1. Our principal result, detailed in the following two theorems, is that the topology $\mathscr{G}$ of $G$ is completely determined by the $\mathscr{G}$-closure $\bar{H}$ of $H$ in $G$.

Theorem 3.2. Let the notation be as in the preceding paragraph. Then

(i) $(G, \mathscr{G})$ has a completion in the category of topological groups.

(ii) $L$ is normal in $G, G=L \bar{H}, L \cap \bar{H}=H$, and $\mathscr{G}=\mathscr{E}\left(\tau, \mathscr{G}_{\bar{H}}\right)$.

(iii) $(G, \mathscr{G})$ is topologically isomorphic with $(L \subseteq \bar{H}) /\left\{\left(h, h^{-1}\right): h \in H\right\}$, 
where the latter has the quotient topology from $\tau \times \mathscr{G}_{\bar{H}}$ and $\bar{H}$ acts on $L$ by inner automorphism.

(iv) If $(L, \tau)$ is $(C A)$, then $\bar{H}$ is the center of $G$.

Theorem 3.3. (i) $(G, \mathscr{G})$ is locally compact, metrizable, complete, or analytic $\Leftrightarrow\left(\bar{H}, \mathscr{G}_{\bar{H}}\right)$ is, respectively, locally compact, metrizable, complete, or Lie.

(ii) If $(G, \mathscr{G})$ is metrizable, then $(G, \mathscr{G})$ has finite topological dimension $\Leftrightarrow\left(\bar{H}, \mathscr{G}_{\bar{H}}\right)$ does.

(iii) $L$ is the $\mathscr{G}$-arc component of $e$ in $G \Leftrightarrow H$ contains the $\mathscr{G}$-arc component of $e$ in $\bar{H}$.

Before proving these theorems, we supplement our introduction with the following remarks about their motivation and context. First we observe that, in the case where $G=L$, both theorems tell us a good deal about the topology of $L$. Second, we point out that Theorem 3.3(iii) can be useful in studying the locally arcwise-connected group associated with $(G, \mathscr{G})$ or some larger group. Third, in response to a suggestion from the referee, we note that, in the analysis of the topology of $\bar{H}$, the closure $\overline{Z(L)}$ of $Z(L)$ may offer a greater challenge than the closure $\bar{V}$ of $V$. Since the adjoint representation maps $V$ injectively into a torus, the choices for the topology $\mathscr{G}_{\bar{V}}$ are limited by the fact that it must be weaker than the usual topology but strong enough to make the mapping of $\bar{V}$ into the torus $\overline{\operatorname{Ad}(V)}$ continuous. On the other hand, the fact that the adjoint representation is trivial on $\overline{Z(L)}$ implies that it can have any (Hausdorff) topology that gives $Z(L)$ a topology weaker than the usual one. Since it is possible, however, for $\bar{H}$ properly to contain $\bar{V} \cdot \overline{Z(L)}$, the analysis of $\bar{H}$ does not reduce simply to the study of the topologies inherited by $\bar{V}$ and $\overline{Z(L)}$. An example where this complication occurs may be found in $\S 6$. Finally, we note that Theorem 3.2(iv) duplicates [4, 4.1(i)] and that portions of Theorems 3.2(ii) and 3.3(i) are similar but not identical to theorems in [4]. This relationship will be more fully explored in $\S 6$.

\section{COMPLETING A (WA) GROUP}

In this section we use the abelian decisive subgroup $H$ to construct a completion of the (WA) group $\left(L, \mathscr{G}_{L}\right)$ and also to prove an important lemma about the completion's topology. We begin by noting that $\left(H, \mathscr{G}_{H}\right)$, as an abelian group, does have a completion $(K, \mathscr{K})$ in the category of topological groups and that $I: H \rightarrow \operatorname{Aut}(L)$, which by Theorem 3.1 is $\mathscr{G}_{H}$-continuous, can therefore be extended to a $\mathscr{K}$-continuous homomorphism $\bar{I}: K \rightarrow \operatorname{Aut}(L)$. Thus the semidirect product $(L(S), \tau \times \mathscr{K})$ is a topological group, and the fact that $\mathscr{K}_{H}=\mathscr{G}_{H}$ is weaker than $\tau_{H}$ implies that $B=\left\{\left(h, h^{-1}\right): h \in H\right\}$ is a closed subgroup. Since $H$ is abelian, it follows that $K$ is abelian and each element of $\bar{I}(K)$ leaves $H$ pointwise fixed. These facts make it easy to verify that $B$ is normal (in fact, central) in $L(S$. Let $M=(L \subseteq K) / B$, with $\mathscr{M}$ the quotient topology and $\pi: L(K \rightarrow M$ the natural projection. The goal of this section is to establish the following theorem.

Theorem 4.1. $(M, \mathscr{M})$ is a completion of $\left(L, \mathscr{G}_{L}\right)$, and the injection $i:\left(L, \mathscr{G}_{L}\right)$ $\rightarrow(M, \mathscr{M})$ is given by $i(x)=\pi(x, e)$ for $x \in L$.

The proof begins with the following lemma, which will also be useful in $\S 5$. 
Lemma 4.2. $L$ contains a subset $C$ with the following properties: (i) $C$ is $\tau$-homeomorphic with a cube in Euclidean space, (ii) the restriction of $\pi$ to $\left(C \times K, \tau_{C} \times \mathscr{K}\right)$ is a homeomorphism onto $\pi(C \times K)$, and (iii) $\pi(C \times K)$ is a complete neighborhood of $e$.

Proof. As a closed subgroup of the analytic group $(L, \tau), H$ has a local crosssection in $L$; that is, $L$ contains a subset $C$ that is $\tau$-homeomorphic with a cube and such that the natural mapping of $(L, \tau)$ onto the set of left cosets $L / H$ is a homeomorphism of $C$ onto a neighborhood of $e$ in $L / H$. (See, e.g., [2, Proposition 1, p. 110].) Therefore $C H$ is a $\tau$-neighborhood of $H$ and the mapping $\varphi: C \times H \rightarrow L$, defined by $\varphi(c, h)=c h$, is, with the relative topologies from $\tau$, a homeomorphism of $C \times H$ onto $C H$. It follows easily that $\pi$ is injective on $C \times K$, and the equality $\pi(C \times K)=\pi((C \times K) B)=\pi(C H \times K)$ implies that $\pi(C \times K)$ is an $\mathscr{M}$-neighborhood of $e$.

To show that $\pi$ is an open mapping of $\left(C \times K, \tau_{C} \times \mathscr{K}\right)$ onto $\pi(C \times K)$, let $(c, k) \in C \times K$ and let $\left\{x_{\alpha}: \alpha \in A\right\}$ be a net in $\pi(C \times K)$ that converges to $\pi(c, k)$. Then there exist a subnet $\left\{x_{\alpha(j)}: j \in J\right\}$ of $\left\{x_{\alpha}\right\}$, a net $\left\{\left(c_{j}, k_{j}\right): j \in\right.$ $J\}$ in $C \times K$, and a net $\left\{\left(h_{j}, h_{j}^{-1}\right): j \in J\right\}$ in $B$ such that $\pi\left(c_{j}, k_{j}\right)=x_{\alpha(j)}$ and $\left(c_{j}, k_{j}\right)\left(h_{j}, h_{j}^{-1}\right)=\left(c_{j} h_{j}, k_{j} h_{j}^{-1}\right) \rightarrow(c, k)$ in $\tau \times \mathscr{K}$. Since $C$ is a local cross-section to $H, c_{j} \rightarrow c$ in $\tau$, and $h_{j} \rightarrow e$ in $\tau$ and thus also in $\mathscr{G}_{H}=\mathscr{K}_{H}$. Therefore $k_{j} \rightarrow k$ in $\mathscr{K}$ and $\pi$ maps $C \times K$ homeomorphically onto $\pi(C \times K)$. In particular, $\pi(e \times K)$ is topologically isomorphic to the complete group $(K, \mathscr{K})$ and, therefore, is complete. To finish the proof of the lemma we note that $\pi(C \times K)$ must, as the product of the compact set $\pi(C \times e)$ and the complete set $\pi(e \times K)$, be complete.

We may now prove Theorem 4.1. By Theorem 3.1(ii) and the definition of "standard extension," the mapping $\gamma: L$ (S) $H \rightarrow L$, defined by $\gamma(x, h)=x h$, is a $\left(\tau \times \mathscr{G}_{H}, \mathscr{G}_{L}\right)$-continuous and open homomorphism with kernel $B$, and thus $i$ is a homeomorphism of $\left(L, \mathscr{G}_{L}\right)$ onto the dense subgroup $(L \mathrm{~S} H) / B$ of $(M, \mathscr{M})$. By [1, Proposition 4, p. 245] and Lemma 4.2, $(M, \mathscr{M})$ is complete and the theorem is proved.

\section{PROOF OF MAIN THEOREMS}

We begin with Theorem 3.2. Adopting the notation of Theorem 4.1, we deduce from Theorem 4.1 and the remark on [8, p. 187] that $(M, \mathscr{M})$ is a completion of $(G, \mathscr{G})$. Thus part (i) is proved, and, as we now show, (ii) follows easily from the identification of $G$ with a dense subgroup of $(M, \mathscr{M})$. Since $i(L)=\pi(L \times H), G$ must have the form $\pi(L \times F)$, where $F$ is a subgroup of $K$ that contains $H$. The normality of $L$ in $G$ is then clear and the homeomorphism of $e \times F$ with $\pi(e \times F)$ that follows from Lemma 4.2 allows us to regard $F$ as a subgroup of $G$, with $\mathscr{G}_{F}=\mathscr{K}_{F}$. Identifying $L$ with $i(L)$, one can easily verify that $G=L F$ and $F \cap L=H$, and the fact that $H$ is $\mathscr{K}$-dense in $K$ implies that $F=\bar{H}$. If we define a mapping $\beta: L(\bar{H} \rightarrow G$ by $\beta(x, f)=x f$, then $\beta$ is a surjective, $\left(\tau \times \mathscr{G}_{\bar{H}}, \mathscr{G}\right)$-continuous homomorphism with kernel $\left\{\left(h, h^{-1}\right): h \in H\right\}$, whence $\beta$ is open and $\mathscr{G}=\mathscr{E}\left(\tau, \mathscr{G}_{\bar{H}}\right)$. This completes the proof of (ii), from which (iii) immediately follows. We conclude the proof by observing that (iv) is an easy consequence of Theorem 3.1(iii) and the equality $G=L \bar{H}$. 
Turning our attention to Theorem 3.3, we note that the $(\Rightarrow)$ portion of each part is trivial. To prove the other half of the theorem, we first apply Lemma 4.2 to obtain a subset $C$ of $L$ that is $\tau$-homeomorphic with a cube and such that the homomorphism $\beta$ in the proof of Theorem 3.2 maps $\left(C \times \bar{H}, \tau \times \mathscr{G}_{\bar{H}}\right)$ homeomorphically onto the $\mathscr{G}$-neighborhood $C \bar{H}$ of $e$. Since $C$ is $\tau$-compact, this homeomorphism implies that $(G, \mathscr{G})$ is locally compact, metrizable, or analytic under the conditions given in (i). To establish the remainder of (i), we simply note that $C \bar{H}$ must be $\mathscr{L}(\mathscr{G})$-complete if $\bar{H}$ is and invoke [1, Proposition 4, p. 245]. Since $\bar{H}$ is $\mathscr{G}$-separable by Theorem 3.1 (iii), (iv), the hypothesis of metrizability in (ii) allows us to use [5, Theorem III.4, p. 33] to finish the proof of the second part of Theorem 3.3. Finally, to prove the $(\Leftarrow)$ part of (iii), let $\lambda:[0,1] \rightarrow G$ be a $\mathscr{G}$-arc with initial point $e$. The homeomorphism of $C \times \bar{H}$ with $C \bar{H}$ provides a lifting $\bar{\lambda}$ of $\lambda$ to a $\tau \times \mathscr{G}_{\bar{H}}$-arc in $L \times \bar{H}$. By hypothesis, $\bar{\lambda}$ lies in $L \times H$ and thus, $\beta(\bar{\lambda})=\lambda$ lies in $L$.

\section{EXAMPLES AND COMPARISON WITH RELATED RESULTS}

According to Theorems 3.2 and 3.3 , the topology of $(G, \mathscr{G})$ is completely determined by its restriction to $\bar{H}$. As we noted in $\S 3$, it is tempting to try to reduce the subsequent investigation of $\mathscr{G}_{\bar{H}}$ to the separate analysis of the topologies of $\bar{V}$ and $\overline{Z(L)}$. Such a reduction is not always possible, however, for $\mathscr{G}_{H}$ might not be a "product topology" (i.e., might not make the projections of $H$ onto $V$ and $Z(L)$ continuous), and in that case $\mathscr{G}_{\bar{H}}$ need not be determined by its restrictions to $\bar{V}$ and $\overline{Z(L)}$. In fact, $\bar{H}=\overline{V \times Z(L)}$ might not, even as a set, equal the product $\bar{V} \cdot \overline{Z(L)}$, as the following example shows. We begin by choosing inductively a sequence $\left\{n_{j}\right\}$ of integers such that, for all positive integers $j, n_{j+1} \geq n_{j}$ ! and the distance between $\exp \left(2 \pi i \sqrt{2} n_{j}\right)$ and 1 is at most $1 / j$. Using [9, Proposition 4.4], we can construct a norm $\mu$ for $\mathbf{R}^{2}$ that is weaker than the usual norm and such that $\mu\left(n_{j}, n_{j}\right) \leq 1 / j$ and $0 \times \mathbf{R}$ inherits the usual topology from $\mu$. By symmetry and the definition of $\mu, \mu$ also induces the usual topology on $\mathbf{R} \times 0$, and it follows that both $\mathbf{R} \times 0$ and $0 \times \mathbf{R}$ are complete subsets of $\left(R^{2}, \mu\right)$. If we associate with each $(a, b) \in \mathbf{R}^{2}$ the homeomorphism of $\mathbf{C}^{2}$ given by

$$
\left(z_{1}, z_{2}\right) \mapsto\left(z_{1} \exp (2 \pi i \sqrt{2} a), z_{2} \exp (2 \pi i a)\right),
$$

then $\left(\mathbf{R}^{2}, \mu\right)$ acts continuously on $\mathbf{C}^{2}$ with the usual topology $\mathscr{C}$ and $\left(\mathbf{C}^{2} \subseteq \mathbf{R}^{2}\right.$, $\mathscr{C} \times \mu)$ is a (WA) group that we will denote by $(L, \mathscr{U})$. It is easy to verify that $H=\mathbf{R}^{2}, V=\mathbf{R} \times 0$, and $Z(L)=0 \times \mathbf{R}$, so that both $V$ and $Z(L)$ are complete in the $\mu$-topology. If $(G, \mathscr{G})$ is the completion of $(L, \mathscr{U})$ and $\bar{H}$ is the $\mathscr{G}$-closure of $H$, then [6, Corollary 6, p. 99] implies that $H$ is a proper subset of $\bar{H}$. On the other hand, since $\bar{V}=V$ and $\overline{Z(L)}=Z(L), \bar{H}$ must properly contain $\bar{V} \cdot \overline{Z(L)}$ and thus $\mathscr{G}_{\bar{H}}$ is not the standard extension of $\mathscr{G}_{\bar{V}}$ and $\mathscr{G}_{\overline{Z(L)}}$ to $\bar{H}$.

Our second example deals with the relationship between our results and those obtained by Goto in [4]. Readers familiar with that paper may have noted that our decisive subgroup $H$ plays a role similar to that of his $g m$-torus, which we will denote here by $J$. In particular, if $\overline{v(J)}$ is the $\mathscr{G}$-closure of the vector part of $J$, then Goto has shown that $G=L \overline{v(J)}$ and that $(G, \mathscr{G})$ is locally 
compact or analytic if and only if $\overline{v(J)}$ is [4, Theorems 3, 5, 6]. Thus $H$ can be replaced by $v(J)$ in some portions of Theorems 3.2 and 3.3, and it is natural to ask whether that substitution is valid in other cases, as well.

Before answering this question, we recall how $J$ and $H$ are defined. Let $\mathbf{l}$ be the Lie algebra of $(L, \tau)$, and let $\overline{\operatorname{Ad}(L)}$ be the closure of the adjoint image of $L$ in the general linear group $\operatorname{GL}(\mathbf{l})$. If $T$ is a maximal torus in $\overline{\operatorname{Ad}(L)}$, then $J=\operatorname{Ad}^{-1}(T)$ is a $\tau$-closed, connected, abelian subgroup of $L$ and, therefore, is the product of a vector group $v(J)$ and a torus [4, 7.2]. To define $H$, we let $N$ be maximal among the connected subgroups of the analytic group $\operatorname{Ad}(L)$ that contain the commutator subgroup of $\operatorname{Ad}(L)$ and are closed in $\overline{\operatorname{Ad}(L)}$. We then choose a torus $S$ that is complementary in $T$ to the torus $N \cap T$ and let $H=\operatorname{Ad}^{-1}(S)$. Then $H=\mathbf{R}^{p} \times T^{q} \times \mathbf{Z}^{r} \times D$, where $\mathbf{R}, T^{q}, \mathbf{Z}$, and $D$ denote, respectively, the real numbers, the $q$-dimensional toroid, the integers, and a finite group, and where $p, q$, and $r$ are nonnegative integers. (Further details about the definition of $H$ can be found in $[9, \S 3]$.) Thus $H$ is always a subgroup of $J$ and $v(J)$ contains the noncompact but not the compact part of $H$.

We may now compare the utility of $H, J, v(J)$, and $\overline{v(J)}$ in describing the topologies of $G$ and $L$. As the example in $[10, \S 8]$ shows, $H$ can be a proper subgroup of $J$ and $\mathscr{G}_{L}$ does not necessarily equal $\mathscr{E}\left(\tau, \mathscr{G}_{v(J)}\right)$, so that $J$ is "too big" and $v(J)$ is "too small" to determine the topology of $L$. It is, however, possible to use the decisiveness of $H$ and the relationship between $H$ and $v(J)$ to show that $\mathscr{G}=\mathscr{E}\left(\tau, \mathscr{G}_{v(J)}\right)$, whence we obtain a topological isomorphism between $(G, \mathscr{G})$ and $(L \mathrm{~S} \overline{v(J)}) /\left\{\left(x, x^{-1}\right): x \in L \cap \overline{v(J)}\right\}$, where the latter has the quotient topology from $\tau \times \mathscr{G}_{\overline{v(J)}}$. As the following example will demonstrate, this representation of $G$ has the disadvantage that $v(J)$ can be a proper subgroup of $L \cap \overline{v(J)}$, so that one must examine the way in which $\overline{v(J)}$ is "glued on" to $L$. Theorem 3.2(iii) shows that this difficulty does not arise if one uses $\bar{H}$ instead of $\overline{v(J)}$.

To illustrate the possibility that $\overline{v(J)}$ contains points of $L$ that are not in $v(J)$, we let $L=\mathbf{R} \times T^{1}$ and $G=L$. Since $L$ is abelian, it follows that $\operatorname{Ad}(L)$ is trivial, $J=H=L$, and $v(J)=\mathbf{R} \times 1$. By 4.3 and 4.5 in [9], there is a topology $\mathscr{U}$ for $L$, weaker than the usual topology, in which the sequence $\left\{\left(n !,(-1)^{n}\right)\right\}$ converges to the identity. Therefore $\{((2 n+1) !, 1)\}$ $\mathscr{U}$-converges to $(0,-1), \overline{v(J)}$ includes $\mathbf{R} \times\{ \pm 1\}$, and $L \cap \overline{v(J)}$ properly contains $v(J)$. (The appropriate choice of $\mathscr{U}$ will ensure that $\overline{v(J)}$ in fact equals $R \times\{ \pm 1\}$.)

\section{A CONJECTURE}

Our principal results can be viewed as structure theorems for groups in which a (WA) group is dense, and we now ask whether any "unexpected" groups fall into this category. If $(A, \mathscr{A})$ is a separable, metrizable group of finite topological dimension and $L$ is the arc-component of the identity in $A$, then [3, Corollary 7.3] implies that $\left(L, \mathscr{A}_{L}\right)$ is a (WA) group. Therefore Theorems 3.2 and 3.3 apply to the $\mathscr{A}$-closure $\bar{L}$ of $L$, and we wish to ask under what circumstances $\bar{L}$ must equal $A$. In response to this question, A. Gleason has 
conjectured that $\bar{L}=A$ if $(A, \mathscr{A})$ is connected and complete. The hypothesis of connectedness is obviously necessary, and the connected group without nontrivial arcs in [7] shows that completeness is necessary as well.

\section{REFERENCES}

1. N. Bourbaki, General topology, Part I, Addison-Wesley, Reading, MA, 1966.

2. C. Chevalley, Theory of Lie groups. I, Princeton Univ. Press, Princeton, NJ, 1946.

3. A. Gleason and R. Palais, On a class of transformation groups, Amer. J. Math. 79 (1957), 631-648.

4. M. Goto, Immersions of Lie groups, J. Math. Soc. Japan 82 (1980), 727-749.

5. W. Hurewicz and H. Wallman, Dimension theory, Princeton Univ. Press, Princeton, NJ, 1948.

6. T. Husain, Introduction to topological groups, W. B. Saunders, Philadelphia, 1966.

7. F. B. Jones, Connected and disconnected plane sets and the functional equation $f(x)+f(y)=$ $f(x+y)$, Bull. Amer. Math. Soc. 48 (1942), 115-120.

8. W. Roelcke and S. Dierolf, Uniform structures on topological groups and their quotients, McGraw-Hill, New York, 1981.

9. T. C. Stevens, Decisive subgroups of analytic groups, Trans. Amer. Math. Soc. 274 (1982), 101-108.

10. __ Weakening the topology of a Lie group, Trans. Amer. Math. Soc. 276 (1983), 541-549.

Department of Mathematics and Computer Science, Saint louis University, Saint LOUIS, MISSOURI 63103

E-mail address: stevensc@sluvca.bitnet 